\title{
(2) OPEN ACCESS \\ Non-surgical animal model of gastroesophageal reflux disease by overeating induced in mice
}

\author{
Nu-Ri IM ๑ , , Byoungjae Kim, ${ }^{1,2}$ Kwang-Yoon Jung, ${ }^{1}$ Tae Hoon Kim, ${ }^{1}$ \\ Seung-Kuk Baek'
}

${ }^{1}$ Department of

Otorhinolaryngology—Head and Neck Surgery, Korea University Medicine, Seoul, South Korea

${ }^{2}$ Neuroscience Research Institute, Korea University Medicine, Seoul, South Korea

Correspondence to Dr Seung-Kuk Baek, Department of Otorhinolaryngology-Head and Neck Surgery, Korea University Medicine, 73 Inchon-ro, Seongbuk-gu, seoul, South Korea: mdskbaek@gmail.com

Accepted 31 March 2021 Published Online First 16 April 2021
Check for updates

(C) American Federation for Medical Research 2022. Re-use permitted under CC BY. Published by BMJ.

To cite: IM N-R, Kim B, Jung K-Y,etal.JInvestigMed 2021;69:1208-1214.

\section{ABSTRACT}

Previous animal models of gastroesophageal reflux disease (GERD) were not physiological and required a variety of surgical procedures. Therefore, the animal model developed by conditions that are similar to the pathogenesis of GERD is necessary. The aim is to establish a non-surgical animal model with GERD caused by overeating induced in mice. To induce mice to overeat, we designed dietary control protocols including repetitive fasting and feeding. The esophageal tissues were evaluated with GERD markers to prove the establishment of a GERD animal model. Mice fasted every other day (group 2) showed more pronounced overeating feature and demonstrated evident changes similar to the macroscopic and microscopic findings of GERD, the expressions of inducible nitric oxide synthase and substance $P$ were stronger. The higher frequency of fasting and overeating could cause GERD effectively. The dietary control can make mice overeat, which elicits the change of lower esophageal mucosa similar to GERD. Thus, the overeating-induced mouse may be used as a GERD mouse model.

\section{INTRODUCTION}

Gastroesophageal reflux disease (GERD) is a digestive disorder caused by acidic gastric juice or food and fluids coming up from the stomach to the esophagus. It is a very common and frequent digestive disorder worldwide. ${ }^{1}$ Approximately half of all adults experience symptoms in their lives. ${ }^{2}{ }^{3}$ Among the various causes, acid and bile juice reflux into the esophageal mucosa are known to be the main causes. ${ }^{45}$ Diverse types of management, such as lifestyle modifications, medications, endoscopic intervention and surgery are adopted for treatment of GERD..$^{6-8}$ Especially, various kinds of medical agents including antacids, histamine 2 receptor antagonists, proton pump inhibitors, transient lower esophageal sphincter relaxation (TLESR) reducer and prokinetics are used for GERD treatment. ${ }^{9}$

The pathogenic mechanisms causing GERD are related to motor abnormalities such as impaired lower esophageal sphincter (LES) resting tone, hernia, visceral hypersensitivity and mucosal resistance damage. ${ }^{10}{ }^{11}$ Most patients with GERD have increased LES relaxation, called TLESR, which is the major

\section{Significance of this study}

What is already known about this subject?

$\Rightarrow$ Overeating in mice can be triggered by repetitive fasting.

$\Rightarrow$ Gastroesophageal reflux is elicited by overeating induced in mice.

$\Rightarrow$ Overeating mouse model without surgical management is a feasible gastroesophageal reflux disease (GERD) model.

What are the new findings?

$\Rightarrow$ GERD can be induced through diet control method in mouse.

$\Rightarrow$ Frequency is more important to trigger GERD than overeating period.

$\Rightarrow$ Overeating induces gastric expansion and causes GERD.

$\Rightarrow \mathrm{A}$ GERD mouse model was created through overeating induced by fasting, which was identified as a GERD disease marker.

How might these results change the focus of research or clinical practice?

$\Rightarrow$ Our findings regarding the non-surgical GERD mouse model is a suitable and physiological model for screening the effects of various drugs on GERD.

$\Rightarrow$ In addition, the result of this study that the frequency of overeating influences disease induction is a clinically meaningful result for the treatment of patients.

pathological mechanism in patients with GERD, accounting for $48 \%-73 \%$ in reflux episodes of GERD symptoms. ${ }^{12-15}$ These TLESRs can be caused by gastric distention, of which especially the subcardiac region is expanded. ${ }^{16}$ According to a previous study, gastric distention in both the normal and the patients with GERD significantly increased the rate of TLESR, and later had affected postprandial increase in gastroesophageal reflux (GER). ${ }^{14}$ For food consumption and gastric distention, various studies have been done, including intakes of diverse meal compositions, ${ }^{1718}$ GERD and meal volume. ${ }^{19}$ Although it is not clear which factor induced the postprandial increase in GERD, it has been 
suggested that gastric distention by food consumption may be a plausible mechanism causing GERD.

An experimental study for GERD using established animal models is important for investigating the therapeutic efficacy and safety of various developed drugs. Although various animals have been used as GERD animal models, most of them required surgical intervention to cause reverse flow, converting gastric or duodenal contents to esophageal. Thus, those surgical models were not a normal physiological condition, and it was difficult to control the amount and concentration of reverse flow. Moreover, postoperative malnutrition and stress made it difficult to analyze data accurately, and most of the animals die quickly because of excessive surgical stress and difficulty in eating. ${ }^{20}$

Therefore, in this study we aim to produce a naturally developed GERD animal model using mice. It was designed to induce overeating for gastric distention without any surgical method under various feeding conditions. Then, we evaluated the suitability of this animal model by examining the expression levels of inducible nitric oxide synthase (iNOS) and substance $\mathrm{P}$ that are representative biomarkers of GERD.

\section{MATERIALS AND METHODS}

\section{Animals}

We obtained C57BL/6 female mice aged 5 weeks from ORIENTBIO (Sungnam, South Korea) and housed them in a specific pathogen-free facility with a 12 hours/ 12 hours light/dark cycle.

\section{Induction of overeating}

We divided the mice for induction of overeating into four groups, as shown in figure 1. Group 1 was a control group with unlimited access to water and food, groups 2-4 were challenge groups with limited access to food. Group 2 fasted every other day, group 3 ate 1 day and fasted two consecutive days and group 4 fasted 1 day after 2 days of eating. We measured body weights and food intake of the mice daily during the experiment. We analyzed the esophagus from mice aged 2, 4 and 8 weeks ( $n=8$ per group).

\section{Histological analysis of esophagus}

We examined esophageal tissues at 2, 4 and 8 weeks after induction of overeating. After we euthanized mice with isoflurane inhalation anesthesia followed by $\mathrm{CO}_{2}$, we excised esophagus tissues and fixed them with $4 \%$ paraformaldehyde. Then, we embedded tissues in paraffin, sectioned them into with $4 \mu \mathrm{m}$ thick slices, stained the slices with H\&E and mounted them on slides.

\section{Immunohistochemistry for GERD markers}

At 8 weeks after induction of overeating, we used immunohistochemistry to detect GERD markers in the esophageal tissues. Archived formalin-fixed, paraffin-embedded tissue samples were paraffin sectioned $(4 \mu \mathrm{m})$, deparaffinized and treated with $0.03 \% \mathrm{H}_{2} \mathrm{O}_{2}$ in methanol for $10 \mathrm{~min}$ to quench endogenous peroxidase activity. After being washed in phosphate-buffered saline (PBS), the sections were then incubated overnight at $4^{\circ} \mathrm{C}$ with iNOS antibody (1:200, Invitrogen, California, USA) or substance P antibody (1:200, Abcam, Cambridge, UK), and were treated with biotinylated antirabbit $\operatorname{IgG}(\mathrm{H}+\mathrm{L})$ or antirat IgG $(\mathrm{H}+\mathrm{L})$ secondary antibody (Vector Laboratories) in PBS (1:400) for 2 hours at room temperature. Then, we detected antigen-antibody complexes using an avidin-biotin complex detection system (Vectastain ABC Kit, Vector Laboratories) and a 3,3'-diaminobenzidine (DAB) Substrate Kit (Vector Laboratories). After counterstaining with Mayer's hematoxylin, we examined the sections using an Olympus BX51 microscope.
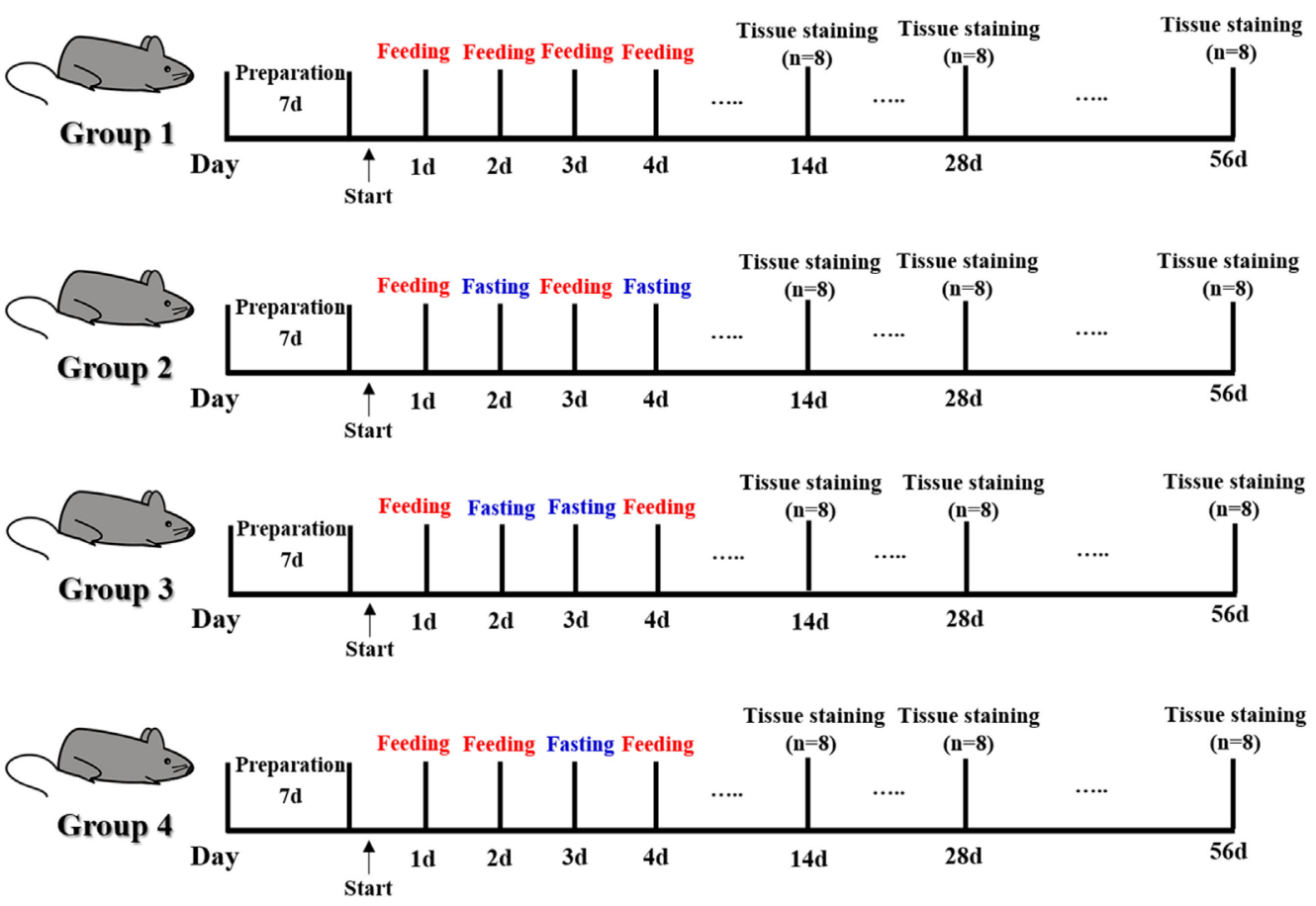

Figure 1 The experimental schemes for various feeding conditions. The figure summarizes four types of schedules on feeding and fasting. 
We captured the pictures in Axio Scan Z1 (ZEISS, Oberkochen, Germany) and compared staining intensity semi-quantitatively using the ImageJ software (National Institutes of Health, Bethesda, USA). Immunostaining of iNOS and substance $\mathrm{P}$ was evaluated in five microscopic fields $(\times 200)$ per tissue of eight different mice in each group. Then, the semi-quantitative score was calculated as the intensity area of the DAB-stained cells in each microscopic field. Then, we classified the stained tissues into a weak intensity group (no or weak stain intensity) and strong intensity group (moderate or strong stain intensity). These results were evaluated by three independent researchers.

\section{Statistics}

We examined the expressions of GERD markers and average food intake per mouse in the experimental groups using the Mann-Whitney $U$ test as appropriate. For all tests, we accepted a probability of $<0.05$ as statistically significant. We did all statistical analyses using SPSS software V.20.0 (IBM SPSS, Armonk, New York, USA).

\section{RESULTS}

\section{Induce overeating and body weight gain through diet}

\section{control}

When we measured body weight and food intake in the control group (group 1), body weight increased steadily during the experimental period, and the daily food intake was not significantly different (figure 2A). In all challenge groups, the body weight decreased after fasting and increased after food intake as expected (figure 2B-D). In groups 2 and 3, the difference of body weight between fasting and food intake was $2-3 \mathrm{~g}$ in average, which was bigger than in group 4 (figure $2 B, C$ ).

To examine the induction of overeating in these challenge groups, we measured food intake on every feeding day. The average food intake per mouse in groups 2 and 3 was about $3 \mathrm{~g}$ more than that in group 1 , whereas the food intake in group 4 was not different from that in group 1 (figure 2E), which indicates that overeating was induced in groups 2 and 3 (figure 2). However, since the overeating frequency of group 2 was higher than that of group 3 but the average body weight of both groups was not different (figure 2B,C,F), we considered that group 2 could be a better model for overeating, a potential GERD trigger (figure 2B,C).

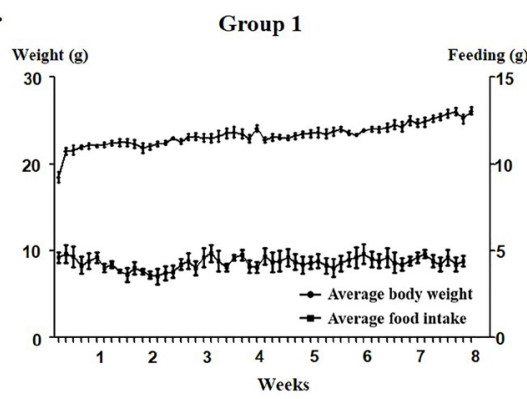

C.

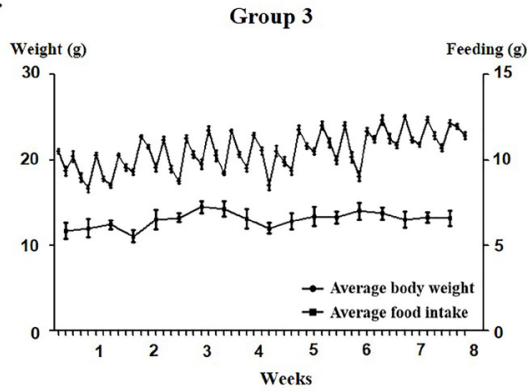

E.

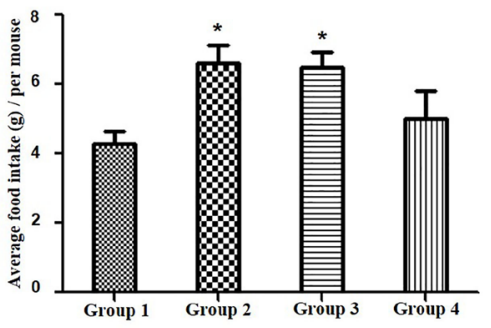

B.

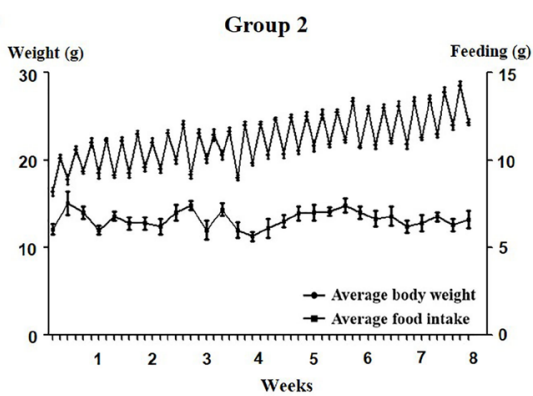

D.

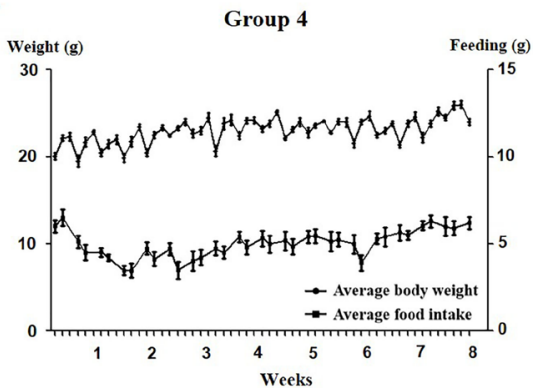

F.

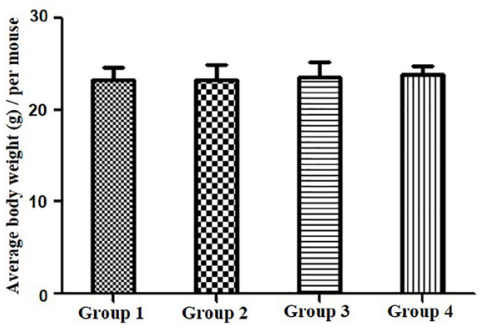

Figure 2 Changes of body weight and food intake in the four experimental groups. (A) Control group without fasting (group 1). (B, C) Group 2 and group 3 showed higher average of food intake and higher fluctuation of body weight than group 1. (D) Group 4 showed no significant difference from group 1. (E) The average food intake per mouse was significantly different for group 2 and group 3 from group 1 $(p<0.05)$. (F) The average body weight per mouse on feeding day was not different among all groups. 
A.

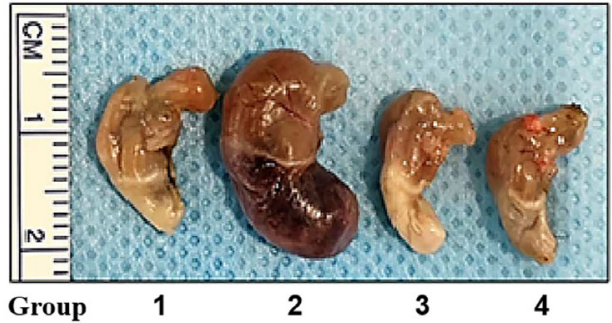

B.

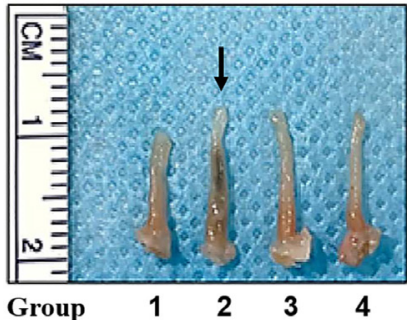

C.

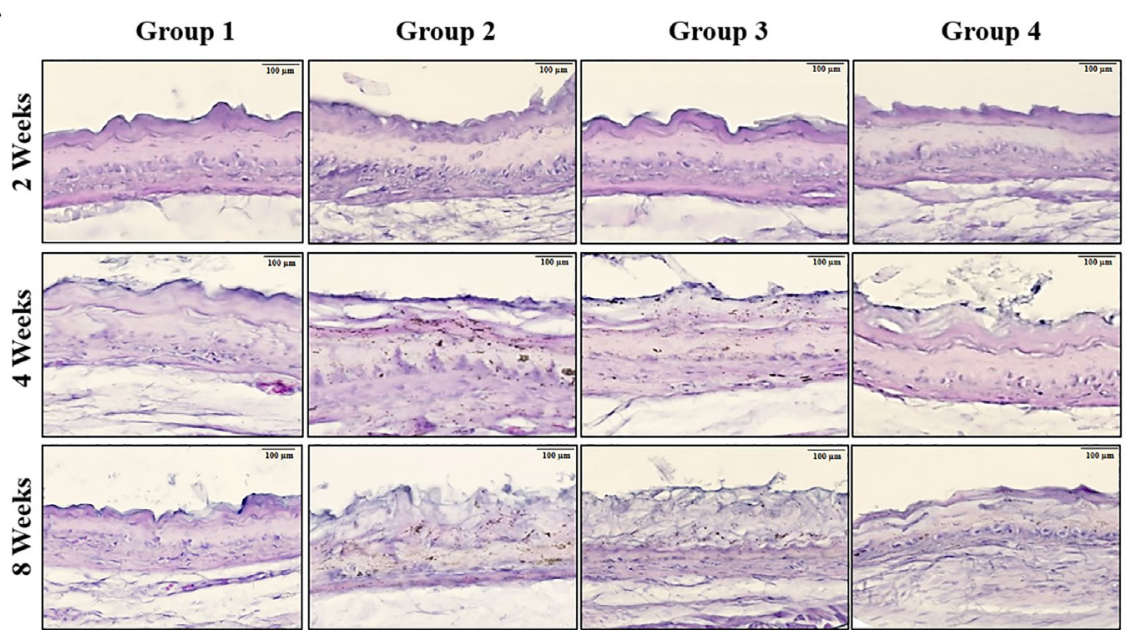

Figure 3 Gross and microscopic change of esophageal inner wall after overeating. (A) Gross appearance of stomach after overeating for 8 weeks. Group 2 showed a larger and engorged stomach compared with the other groups. (B) Gross appearance of esophagus in 8 weeks of overeating. Group 2 showed more engorged esophagus (arrow). (C) The H\&E staining of lower esophageal tissues from each group. In group 2, the mucosal layer of the esophagus was most damaged in 8 weeks (bar, $100 \mu \mathrm{m})$.

\section{Macroscopic appearances of stomach and esophagus and histological changes in the lower esophageal mucosa of the overeating-induced mice}

We examined the gross appearances of the stomach and esophagus at 8 weeks of the experiment. The stomach of group 2 was more distended and engorged than were those in the control group and other challenge groups, this same result showed seven mice of eight mice in group 2, which may mean that sufficient overeating was elicited in group 2 (figure $3 \mathrm{~A}$ ). Furthermore, discoloration of esophageal tissue was shown in group 2, not in other groups, suggesting that the overeating could induce some damages to esophageal tissue (figure 3B).

After 2 weeks of feeding control, the histological changes of the esophagus in the challenge groups were not examined compared with the control group. In groups 2 and 3, the cellular debris-like particles were elicited within the mucosal layer of the esophagus from week 4 of the experiment and then, the keratin layers of the esophagus were disorganized and exfoliated at week 8 of the experiment. In particular, such a gross change of esophageal mucosa was more definite in group 2, suggesting that overeating can cause mucosal changes in the lower esophageal epithelium (figure 3C).

\section{Expression of iNOS and substance $P$ in lower esophageal mucosa}

To evaluate whether GERD occurred in the overeating model, we stained the lower esophageal mucosa with
iNOS and substance P, known as GERD markers. Among three challenge groups, the expressions of iNOS and substance $P$ was strongest in group $2(p<0.05$, table 1$)$ (figure 4).

\section{DISCUSSION}

This is the first experimental report to confirm a link between GERD and overeating in the non-surgical animal model. We generated an overeating mouse model by using various dietary controls, including fasting and

Table 1 Expressions of iNOS and substance P according to the experimental groups

\begin{tabular}{|llllll|}
\hline Markers & IHC score & Group 1 (\%) & Group 2 (\%) & Group 3 (\%) & Group $4(\%)$ \\
\hline iNOS & & & & & \\
\hline & Weak & $8(100)$ & $2(25)$ & $3(60)$ & $4(50)$ \\
\hline & Strong & $0(0)$ & $6(75)$ & $5(40)$ & $4(50)$ \\
\hline Substance P & P value & & 0.007 & 0.026 & 0.077 \\
\hline & Weak & $8(100)$ & $1(12.5)$ & $2(25)$ & $4(50)$ \\
\hline & Strong & $0(0)$ & $7(87.5)$ & $6(75)$ & $4(50)$ \\
& P value & & 0.001 & 0.007 & 0.077
\end{tabular}

Staining intensity scores was as follows: weak=no staining and mild stain intensity, strong=moderate and strong stain intensity. We did statistical analysis using SPSS V.20.0 (IBM, Armonk, New York, USA). Statistical significance was set at $\mathrm{p}<0.05$.

IHC, immunohistochemical stain; iNOS, inducible nitric oxide synthase. 

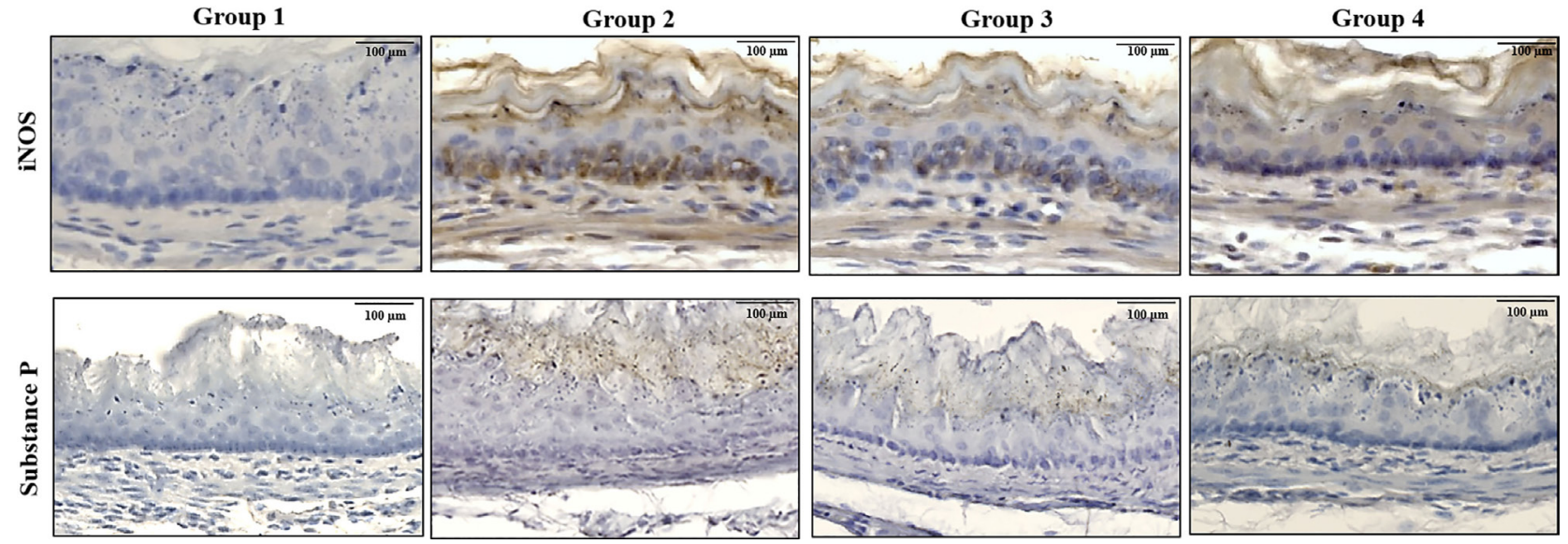

Figure 4 Expressions of inducible nitric oxide synthase (iNOS) and substance $P$ in the immunohistochemical staining of esophageal mucosa. In 8 weeks of experiment, the expression of iNOS and substance P were significantly higher in overeating-induced groups (groups 2-4) compared with the control group (group 1). Especially, group 2 showed the strongest expressions of iNOS and substance P.

feeding, and demonstrated that overeating can lead to GERD that is physiologically induced without any surgery, by identifying that overeating causes significant damage and overexpression of GERD markers in the lower esophageal mucosa.

In the patients with GERD, increased TLESR or hypotensive LES is usually accompanied by dysfunction of the esophagogastric junction (EGJ), which allows more gastric contents to reflux into the esophagus and result in reflux esophagitis. $^{21-23}$ Because the regulation of EGJ pressure is mainly achieved by LES, ${ }^{24}$ a weakened antireflux barrier at the EGJ by gastric distention may be related to increased TLESR and its GERD pathogenesis. ${ }^{25-27}$ Considering such a pathogenesis of GERD, we applied various frequencies of fasting and feeding to mice, and confirmed that the dietary control can induce mice to overeat in this study. This overeating at 8 weeks caused gastric distention and esophageal injury by frequent reflux of gastric contents. Among various dietary controls, the fasting every other day (group 2) was more effective in eliciting inflammation and damage of the lower esophageal mucosa than was fasting for a longer duration. The food intake and body weight in the mice of group 2 were not different from those of the normal control group (group 1), which suggests the well-being of the mice. Furthermore, the macroscopic and microscopic examinations of the esophagus in mice in which GERD was induced by overeating showed damaged mucosal barrier and overexpression of iNOS and substance $\mathrm{P}$ in the lower esophageal region. Especially, such mucosal changes of esophageal inner epithelium were more severe in group 2, with fasting every other day, than in the other groups. Thus, the most effective dietary control to induce GERD was the fasting every other day for at least 8 weeks, which means that a higher frequency of fasting and overeating is important in the pathogenesis of GERD.

The previous studies have suggested that iNOS is induced by reflux components like stomach acid or bile acids in esophageal cells. ${ }^{5} 28$ The expression of iNOS was also induced in the esophagus in an experimental rat model eliciting duodenal reflux. ${ }^{30}$ Especially, iNOS was increased in human biopsies from inflamed esophageal mucosa of GERD, Barrett's esophagus or patients with esophageal adenocarcinoma. The overexpression of iNOS in these tissues showed increasing frequency, suggesting that it is an active inflammatory process in the reflux disease spectrum and is a result of GERD. ${ }^{31}$ In addition, other studies have demonstrated that substance $\mathrm{P}$ overexpresses in esophageal reflux disease results from activation of reflux-related receptors, transient receptor potential vanilloid receptor 1 or protease-activated receptor 2 , resulting in heart burn or esophageal edema. ${ }^{32} 33$ The expression of substance $P$ and acid reflux score had positive correlation and the substance $\mathrm{P}$ was immunostained with the nerve fibers in esophageal mucosa. ${ }^{34}$ Therefore, based on those previous studies, we evaluated the expressions of iNOS and substance P as GERD markers in the esophageal epithelium, and proved that the expressions of these markers are increased, which presented biochemical proof of GERD development in the mouse model.

Gender is one of the important factors for establishing animal models because the reactions to drugs and incidence and progression of diseases, is different depending on genders. In our research, there are two reasons for using female mice to develop a GERD nonsurgical animal model. First, according to the previous study, gender is one of the potential risk factors of GERD because the incidence and symptoms is high in women. ${ }^{35-37}$ Understanding these gender-related biological factors is important for the future prevention and treatment of GERD. Second, previous study suggested that gender is regarded as one of the inducing factors for obesity. As a result of long-term observation, male mice showed a significantly difference in body weight when feeding a high-fat diet and a low-fat diet while the female mice was not. This result indicates that the female is not suitable for obesity-inducing models. ${ }^{38}$ Therefore, our study used female mice because it aims to induce GERD disease just by regulating the frequency and duration of food intake, not by inducing obesity or weight gain with different kind of food.

The limitation of this present study is that direct evidence that overeating elicits the reflux of gastric content to the esophagus could not be suggested. Although we demonstrated the macroscopic and microscopic changes of the 
lower esophageal mucosa, a future study may be necessary to prove the evidence of hypotensive LES and esophageal reflux. However, since the animal model of this study is elicited by relatively physiological conditions, various traditional or promising drugs that are associated with GERD or laryngopharyngeal reflux disease may be easily evaluated for their effectiveness and safety.

In conclusion, in this study we suggested a GERD mouse model using a non-surgical method. Overeating, one of the main causes of GERD, may be induced by various dietary controls, including fasting and feeding, in mice, and a higher frequency of fasting and overeating could cause GERD effectively. In addition, the establishment of the GERD animal model could be confirmed by the overexpression of iNOS and substance P in esophageal mucosa. Therefore, we suggest that this overeating-induced by fasting can be used as a GERD mouse model.

Contributors N-RIM: designed the study, developed the methodology, acquired data and wrote and reviewed the manuscript. BK: drafted the study, collecting, interpreting data and revision of the manuscript. K-YJ, THK: collected and interpreted data. S-KB: designed the study, wrote and reviewed the manuscript. N-RIM, BK, K-YJ, THK, S-KB: approved the final draft of the submitted manuscript.

Funding This study was supported by the Clinical Trial Center of Korea University Anam Hospital (11502411), the Korea Health Technology R\&D Project (H14C0748) through the Korea Health Industry Development Institute (KHIDI) by the Ministry of Health \& Welfare and the Basic Science Research Program through the National Research Foundation of Korea (NRF) funded by the Ministry of Education (NRF2018R1D1A1A09083263).

\section{Competing interests None declared.}

Patient consent for publication Not required.

Ethics approval Protocols and experimental design parameters were reviewed and approved by the institutional Review Board of Korea University Hospital (IRB No. KOREA-2018-0026). All experiments were done in accordance with relevant guidelines and regulations.

Provenance and peer review Not commissioned; externally peer reviewed. Data availability statement All data relevant to the study are included in the article or uploaded as supplementary information.

Open access This is an open access article distributed in accordance with the Creative Commons Attribution 4.0 Unported (CC BY 4.0) license, which permits others to copy, redistribute, remix, transform and build upon this work for any purpose, provided the original work is properly cited, a link to the licence is given, and indication of whether changes were made. See: https:// creativecommons.org/licenses/by/4.0/.

\section{ORCID iD}

Nu-Ri IM http://orcid.org/0000-0001-6827-7354

\section{REFERENCES}

1 Robinson M, Maton PN, Allen ML, et al. Effect of different doses of omeprazole on 24-hour oesophageal acid exposure in patients with gastro-oesophageal reflux. Aliment Pharmacol Ther 1991;5:645-51.

2 Mosli M, Alkhathlan B, Abumohssin A, et al. Prevalence and clinical predictors of Ipr among patients diagnosed with GERD according to the reflux symptom index questionnaire. Saudi J Gastroentero/ 2018;24:236-41.

3 Cohen E, Bolus R, Khanna D, et al. Gerd symptoms in the general population: prevalence and severity versus care-seeking patients. Dig Dis Sci 2014;59:2488-96.

4 Wang R-H. From reflux esophagitis to Barrett's esophagus and esophageal adenocarcinoma. World J Gastroenterol 2015;21:5210-9.

5 McAdam E, Haboubi HN, Forrester $\mathrm{G}$, et al. Inducible nitric oxide synthase (iNOS) and nitric oxide (NO) are important mediators of reflux-induced cell signalling in esophageal cells. Carcinogenesis 2012;33:2035-43.

6 Labenz J, Malfertheiner P. Treatment of uncomplicated reflux disease. World J Gastroenterol 2005;11:4291-9.
7 Corley DA, Katz P, Wo JM, et al. Improvement of gastroesophageal reflux symptoms after radiofrequency energy: a randomized, sham-controlled trial. Gastroenterology 2003;125:668-76.

8 Allaix ME, Herbella FA, Patti MG. Laparoscopic total fundoplication for gastroesophageal reflux disease. How I do it. J Gastrointest Surg 2013;17:822-8

9 Sandhu DS, Fass R. Current trends in the management of gastroesophageal reflux disease. Gut Liver 2018;12:7-16.

10 De Giorgi F, Palmiero M, Esposito I, et al. Pathophysiology of gastrooesophageal reflux disease. Acta Otorhinolaryngol Ital 2006;26:241-6.

11 Böhmer AC, Schumacher J. Insights into the genetics of gastroesophageal reflux disease (GERD) and GERD-related disorders. Neurogastroenterol Motil 2017;29:e13017.

12 Dent J, Dodds WJ, Friedman RH, et al. Mechanism of gastroesophageal reflux in recumbent asymptomatic human subjects. J Clin Invest 1980;65:256-67.

13 Massey BT, Simuncak C, LeCapitaine-Dana NJ, et al. Transient lower esophageal sphincter relaxations do not result from passive opening of the cardia by gastric distention. Gastroenterology 2006;130:89-95.

14 Holloway RH, Hongo M, Berger K, et al. Gastric distention: a mechanism for postprandial gastroesophageal reflux. Gastroenterology 1985;89:779-84.

15 Mittal RK, McCallum RW. Characteristics and frequency of transient relaxations of the lower esophageal sphincter in patients with reflux esophagitis. Gastroenterology 1988;95:593-9.

16 Halicka J, Banovcin P, Halickova M, et al. Acid infusion into the esophagus increases the number of meal-induced transient lower esophageal sphincter relaxations (TLESRs) in healthy volunteers. Neurogastroenterol Motil 2014:26:1469-76.

17 Nebel OT, Castell DO. Lower esophageal sphincter pressure changes after food ingestion. Gastroenterology 1972;63:778-83.

18 Ledeboer M, Masclee AA, Batstra MR, et al. Effect of cholecystokinin on lower oesophageal sphincter pressure and transient lower oesophageal sphincter relaxations in humans. Gut 1995;36:39-44.

19 Hargrave SL, Kinzig KP. Repeated gastric distension alters food intake and neuroendocrine profiles in rats. Physiol Behav 2012;105:975-81.

20 Kapoor H, Lohani KR, Lee TH, et al. Animal models of Barrett's esophagus and esophageal adenocarcinoma - past, present, and future. Clin Trans/ Sci 2015;8:841-7.

21 Lin S, Li H, Fang X. Esophageal motor dysfunctions in gastroesophageal reflux disease and therapeutic perspectives. J Neurogastroenterol Motil 2019;25:499-507.

22 Gyawali CP, Roman S, Bredenoord AJ, et al. Classification of esophageal motor findings in gastro-esophageal reflux disease: conclusions from an international consensus group. Neurogastroenterol Motil 2017;29. doi:10.1111/nmo.13104. [Epub ahead of print: 24 May 2017].

23 Sun X, Ke M, Wang Z, et al. [Characteristics of esophageal motility in patients with non-erosive reflux disease and reflux esophagitis]. Zhonghua Yi Xue Za Zhi 2014;94:1718-21.

24 Beaumont $\mathrm{H}$, Bennink RJ, de Jong J, et al. The position of the acid pocket as a major risk factor for acidic reflux in healthy subjects and patients with GORD. Gut 2010;59:441-51.

25 Fock KM, Talley N, Goh KL, et al. Asia-Pacific consensus on the management of gastro-oesophageal reflux disease: an update focusing on refractory reflux disease and Barrett's oesophagus. Gut 2016:65:1402-15.

26 Eslami 0, Shahraki M, Bahari A, et al. Dietary habits and obesity indices in patients with gastro-esophageal reflux disease: a comparative cross-sectional study. BMC Gastroenterol 2017;17:132.

27 Savarino E, Bredenoord AJ, Fox M, et al. Advances in the physiological assessment and diagnosis of GERD. Nat Rev Gastroenterol Hepatol 2018;15:323.

28 Jiang B, Zhao S, Tao Z, et al. Controlled bile acid exposure to oesophageal mucosa causes up-regulation of nuclear $\gamma$-H2AX possibly via iNOS induction. Biosci Rep 2016;36. doi:10.1042/BSR20160124. [Epub ahead of print: 07 Jul 2016].

29 Feagins LA, Zhang HY, Zhang $X$, et al. Mechanisms of oxidant production in esophageal squamous cell and Barrett's cell lines. Am J Physiol Gastrointest Liver Physiol 2008;294:G411-7.

30 Bae JD, Jung KH, Ahn WS, et al. Expression of inducible nitric oxide synthase is increased in rat Barrett's esophagus induced by duodenal contents reflux. J Korean Med Sci 2005:20:56-60.

31 Vaninetti NM, Geldenhuys L, Porter GA, et al. Inducible nitric oxide synthase, nitrotyrosine and p53 mutations in the molecular pathogenesis of Barrett's esophagus and esophageal adenocarcinoma. Mol Carcinog 2008:47:275-85

32 Yoshida N. Inflammation and oxidative stress in gastroesophageal reflux disease. J Clin Biochem Nutr 2007:40:13-23. 
33 Vergnolle N, Bunnett NW, Sharkey KA, et al. Proteinase-activated receptor-2 and hyperalgesia: a novel pain pathway. Nat Med 2001;7:821-6.

34 Yoshida N, Kuroda M, Suzuki T, et al. Role of nociceptors/neuropeptides in the pathogenesis of visceral hypersensitivity of nonerosive reflux disease. Dig Dis Sci 2013;58:2237-43.

35 Fakhre Yaseri H. Gender is a risk factor in patients with gastroesophageal reflux disease. Med J Islam Repub Iran 2017:31:336-8.
36 Asanuma K, lijima K, Shimosegawa T. Gender difference in gastro-esophageal reflux diseases. World I Gastroenterol 2016;22:1800-10.

37 Kim YS, Kim N, Kim GH. Sex and gender differences in gastroesophageal reflux disease. J Neurogastroenterol Motil 2016;22:575-88.

38 Yang Y, Smith DL, Keating KD, et al. Variations in body weight, food intake and body composition after long-term high-fat diet feeding in C57BL/6J mice. Obesity 2014;22:2147-55. 